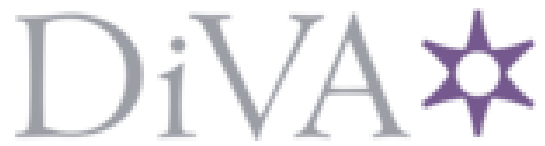

http://www.diva-portal.org

Postprint

This is the accepted version of a paper presented at International Conference on Pattern Recognition, ICPR.

Citation for the original published paper:

Ringqvist, C., Butepage, J., Kjellström, H., Hult, H. (2020)

Interpolation in Auto Encoders with Bridge Processes

In:

https://doi.org/10.1109/ICPR48806.2021.9413123

N.B. When citing this work, cite the original published paper.

Permanent link to this version:

http://urn.kb.se/resolve?urn=urn:nbn:se:kth:diva-295217 


\title{
Interpolation in Auto Encoders with Bridge Processes
}

\author{
Carl Ringqvist, Judith Butepage, Hedvig Kjellström, and Henrik Hult
}

\begin{abstract}
Auto encoding models have been extensively studied in recent years. They provide an efficient framework for sample generation, as well as for analysing feature learning. Furthermore, they are efficient in performing interpolations between datapoints in semantically meaningful ways. In this paper, we introduce a method for generating sequence samples from auto encoders trained on flattened sequences (e.g video sample from auto encoders trained on single frames); as well as a canonical, dimension independent method for generating stochastic interpolations. The distribution of interpolation paths is represented as the distribution of a bridge process constructed from an artificial random data generating process in the latent space, having the prior distribution as its invariant distribution.
\end{abstract}

\section{Introduction}

Consider a data set $\left\{x^{(i)}\right\}_{i=1}^{N}$ generated from an unknown probability $p$ on $\mathbb{R}^{d}$. Interpolation between two data-points $x^{(i)}$ and $x^{(j)}$ aims at generating semantically meaningful transition paths from $x^{(i)}$ to $x^{(j)}$. Examples include smooth realistic transitions between images, imputation of missing data, generation of time-series from snapshots, etc. If the dimension $d$ is large and $p$ is mainly concentrated near a low-dimensional manifold, then the interpolation path must reside near that manifold and avoid regions where $p$ places insignificant mass.

Learning a representation of the data is useful in this context, as the representation may be used to understand the underlying geometry and characterize interpolation paths. More specifically, we are concerned with the construction of a probability distribution over interpolation paths, and algorithms for sampling from that distribution. Sampling random interpolation paths is useful for generating video from snapshots, motion from static positions, etc.

KTH Royal Institute of Technology, Lindstedtsvagen 25, 100 44, Stockholm, Sweden. e-mail: carrin@kth.se, e-mail: butepage@kth.se, e-mail: hedvig@kth.se, e-mail: hult@kth.se 
To model the distribution over interpolation paths it is convenient to consider an artificial data generating mechanism, in the form of a stationary stochastic process, $\{\mathbf{X}(t)\}_{t \in[0, T]}$ having $p$ as its invariant distribution and consider the distribution of the corresponding bridge process, obtained by conditioning $\mathbf{X}$ on its start and end points, $\mathbf{X}(0)=x^{(i)}$ and $\mathbf{X}(T)=x^{(j)}$. The properties of the interpolation paths will inherently depend on the properties of the underlying stochastic process $\mathbf{X}$ and the length of the time interval, $T$.

Stochastic processes that represent artificial data generating mechanisms are frequently encountered in the context of manifold learning. The data distribution $p$ is closely related to the invariant distribution of a reflected diffusion process on a compact domain, constructed as the limit of a random walk on an embedded graph, see e.g. [24, 25, 15] and [9].

In this paper, the Variational Auto Encoder (VAE) will be employed to model the data distribution $p$, see [20]. The VAE learns a distribution $p(x)$ over static data $\left\{x^{(i)}\right\}_{i=1}^{N}$ through the use of an imposed latent variable $Z$. It consists of an encoder, $q_{\phi}(z \mid x)$, a prior distribution, $p(z)$, and a decoder, $p_{\theta}(x \mid z)$. VAEs have been applied to several challenging data sets to extract meaningful representations. Various extensions of VAEs have achieved state-of-the-art performance in hierarchical organization of latent spaces, disentanglement and semi-supervised learning [30]. The auto encoding model structure has many desirable properties. For example, it enables an easy mechanism for generating samples from the data distribution through sampling $x \sim p_{\theta}(x \mid z)$, where $z \sim p(z)$. The simple structure of the prior distribution facilitates different types of data analysis such as clustering. Furthermore, the model setting has effective interpolation capabilities. Interpolation between two data-points $x^{(i)}$ and $x^{(j)}$ can be performed with a trained VAE according to Algorithm 1 below. To reduce noise, the sampling steps (1) and (3) are often replaced by taking means. If the latent path is properly constructed, the output transformation can be semantically meaningful. To construct a stochastic interpolation step (2) is replaced by considering the bridge process $\left\{\mathbf{Z}^{(i j)}(t)\right\}_{t \in[0, T]}$ from $z^{(i)}$ to $z^{(j)}$ associated with a stochastic process $\{\mathbf{Z}(t)\}_{t \in[0, T]}$ with invariant distribution $p(z)$, by conditioning

Algorithm 1 Constructing latent interpolations

1. Encode $x^{(i)}, x^{(j)}$ through sampling $z^{(i)} \sim q_{\phi}\left(z \mid x^{(i)}\right), z^{(j)} \sim q_{\phi}\left(z \mid x^{(j)}\right)$

2. Construct a suitable path in the latent space between the decoded points $\left[z^{(i)}, z, \ldots, z^{(j)}\right]$

3. Decode the latent path by sampling

$$
\left[x^{(i)} \sim p_{\theta}\left(x \mid z^{(i)}\right), x \sim p_{\theta}(x \mid z), \ldots, x^{(j)} \sim p_{\theta}\left(x \mid z^{(j)}\right)\right]
$$

on $\mathbf{Z}(0)=z^{(i)}$ and $\mathbf{Z}(T)=z^{(j)}$. The corresponding stochastic interpolation from $x^{(i)}$ to $x^{(j)}$ is given by the bridge process $\left\{\mathbf{X}^{(i j)}(t)\right\}_{t \in[0, T]}$ where $\mathbf{X}^{(i j)}(0)=x^{(i)}$, 
$\mathbf{X}^{(i j)}(T)=x^{(j)}$, and

$$
\mathbf{X}^{(i j)}(t)=\int x p_{\theta}\left(x \mid \mathbf{Z}^{(i j)}(t)\right) d x .
$$

The proposed methodology for stochastic interpolation can easily be adapted to generate semantically meaningful random sequences of synthetic data of arbitrary length, from a given starting point, $x_{0}$. Such sequences, $\{\mathbf{X}(t)\}_{t \geq 0}$ are obtained by simply encoding $x_{0}$ by sampling $z_{0} \sim q_{\phi}\left(z \mid x_{0}\right)$, sampling the stochastic process $\{\mathbf{Z}(t)\}_{t \geq 0}$ conditional on $\mathbf{Z}(0)=z_{0}$ and decoding

$$
\mathbf{X}(t)=\int x p_{\theta}(x \mid \mathbf{Z}(t)) d x .
$$

The paper is organised as follows. In Section 2 related work on manifold learning, deterministic interpolation and stochastic process VAEs is presented. Section 3 contains background on bridge processes constructed from diffusions and Gaussian processes. The main results, presented in Section 4, provide theoretical evidence that the produced interpolations respects the distribution of the data, and indicate our method in some sense can be viewed as a generalisation of both linear and spherical interpolation. Numerical experiments are provided in Section 5.

To summarize, the main contributions of this paper are:

1. We propose a method for generating random interpolation paths based on bridge processes. To our knowledge, previously considered interpolation methods are deterministic, but the random property is interesting and desirable for some applications.

2. As already indicated, the methodology can be used for generating semantically meaningful sample sequences from models trained on independent and identically distributed data (e.g video samples from VAEs trained on iid image frames).

3. We demonstrate, theoretically and empirically, that the proposed interpolation method has the desirable property of producing interpolations respecting the distribution of the data, regardless of dimension.

4. We demonstrate for that for normal priors, our interpolation method produces a stochastic generalisation of both linear interpolation and spherical interpolation.

\section{Related work}

Construction of deterministic interpolation paths that take the data distribution into account relies on finding geodesics in appropriate metrics. A natural approach is to consider geodesic distances based smooth paths $\gamma$ that minimize a cost functional of the form $\int_{0}^{1} f(\gamma(t), \dot{\gamma}(t)) d t$. Such metrics may be obtained by constructing 
neighborhood graphs from an iid sample of size $n$ according to some density $p$, with edge weights as a function of the distance, and consider the limit of the graph distance as $n \rightarrow \infty$. This approach includes the $f$-geodesics by [2], the directed embeddings of [24, 25], and the hitting-time approach of [15], see also [9] and the references therein for the general setting.

In the context of VAEs, which are usually trained by maximising a variational lower bound of the log-likelihood, encoded data-points will typically reside in areas of high prior distribution mass, and the decoder will produce meaningful output only from these areas. If an interpolation path is constructed without taking the prior distribution into consideration, intermediate points may be placed where the prior distribution has low mass, resulting in data samples of low fidelity. For example, if the prior is chosen to be a standard normal of high dimension, codes tend to lie near a sphere around the origin with radius given by the square-root of the dimension of the latent space [14]. Taking, say, a linear path between two encoded data-points will thus traverse through the interior of the sphere where the prior distribution mass may be low.

Various methods for dealing with the prior/path relationship have been proposed. In [31], a remedy to the problem encountered in the aforementioned example with spherically concentrated codes is proposed by considering spherical interpolation. Although improvements in sample quality is observed, it gives no indication on the threshold at which it is suitable to switch from linear to spherical interpolation. Furthermore, the method does not generalise to arbitrary prior distributions. In [1], an interpolation method is suggested that is applicable to a general class of prior distributions. It is using the distributional mismatch of linearly interpolated points as starting point, and aims to construct paths of lesser mismatch. A warping technique using optimal transport is considered, where an interpolation along an 'intuitive' path (e.g linear) is transformed into nearby path with interpolation points following the prior distribution. The distributional mismatch of linearly interpolated points as observed in [19] have been the basis of several additional suggestions for suitable interpolation paths. In [19], a prior distribution is suggested on basis of exhibiting lower distribution mismatch. Here, prior samples are generated uniformly from the sphere, but adjusted so that the sample radius follows a gamma distribution. Intuitively, the scheme tends to place prior samples inside the sphere to a much larger extent than does e.g. the normal prior of high dimension; thus reducing the problem of void linear paths between sampled points. In [22], is shown that a Cauchy prior produces a Cauchy distributed midpoint when sampling endpoints from the Cauchy prior, thus exhibiting perfect distributional match between the sampled end-points and the midpoint of a linear interpolation. However, the Cauchy distribution has undefined moments and heavy tails, causing problems with extreme sample values and making analysis hard. In [27], a prior exhibiting low midpoint distributional mismatch is searched for and obtained through numerical optimisation. The resulting prior has low midpoint distributional mismatch, bounded domain and finite moments. Although the distributional 
mismatch formulation is a good illustrator of the problems with interpolations not respecting the prior format, it has some shortcomings serving as optimisation criteria for suitable paths. An interpolation scheme based on low interpolation distributional mismatch will only offer a solution that is good on average, which is a weak form of optimality.

In [4], an entirely different and interesting approach is considered. The method suggests encouraging interpolation quality through including an interpolation quality critic network in the training process. The method is applied to a vanilla auto-encoder, as such imposing no structure whatsoever on the prior distribution. Although visual inspection improvements are obtained, the method is deprived of a valuable tool for analysing the latent variable manifold.

There have been attempts to use VAEs for dependent data. In [6], Gaussian processes are considered as explicit priors in VAEs where the covariance function of the Gaussian process aims to capture the dependence structure in the training data. The authors develop the variational inference approach to train such models. Related work include the use of recurrent network encoders and decoders for representation learning, classification and forecasting [28, 16, 23].

In a similar direction [13] propose a VAE architecture for multivariate time series imputation with a Gaussian process prior in the latent space to capture temporal dynamics. Stochastic process VAEs are further developed in the $\mathrm{ODE}^{2} \mathrm{VAE}$ model [32] that introduce a model that can simultaneously learn the embedding of high dimensional trajectories and infer complex continuous-time latent dynamics.

Although there are close connections to stochastic process VAEs, we emphasize that in this paper independence between data points is assumed and hence the dependence cannot be inferred from the data. In fact, any latent stochastic processes with the prior as its invariant distribution may be used for stochastic interpolation and every such choice will lead to a different interpolation scheme, via the associated bridge process.

Our approach is different from previous approaches to interpolation, mentioned above, which rely on deterministic methods. We introduce stochastic interpolation by representing the distribution of interpolation paths in the form of associated stochastic bridge processes. There are certainly connections both to manifold learning and to VAEs for dependent data, but none of these existing approaches have been used to study interpolation.

\section{Diffusions, Gaussian processes and bridges}

Let $\mathbf{X}=\{\mathbf{X}(t)\}_{t \in[0, T]}$ be a stochastic process in $\mathbb{R}^{d}$ and $\left\{\mathbf{X}^{x_{0} x_{T}}(t)\right\}_{t \in[0, T]}$ be the corresponding bridge process, obtained by conditioning $\mathbf{X}$ on its start and end points, $\mathbf{X}(0)=x_{0}$ and $\mathbf{X}(T)=x_{T}$. In the following, bridge processes constructed from diffusions and Gaussian processes will be considered. 


\subsection{Diffusion bridges and the Ornstein-Uhlenbeck process}

Consider a diffusion process $\mathbf{X}=\{\mathbf{X}(t)\}_{t \in[0, T]}$ with values in $\mathbb{R}^{d}$ satisfying the stochastic differential equation $(\mathrm{SDE})$

$$
d \mathbf{X}(t)=\mu(\mathbf{X}(t)) d t+\sigma(\mathbf{X}(t)) d \mathbf{W}(t)
$$

where $\mathbf{W}$ is a Brownian motion and the drift $\mu$ and diffusion coefficient $\sigma$ satisfy appropriate conditions so that a strong solution exists, see e.g. [18, Ch. 5.2]. Given a probability $p$ of the form $p(\mathbf{x}) \propto e^{-E(\mathbf{x})}$ a Langevin diffusion of the form

$$
d \mathbf{X}=-\nabla E(\mathbf{X}) d t+\sqrt{2} d \mathbf{W}
$$

has $p$ as its stationary distribution (under suitable conditions). The corresponding bridge process, $\mathbf{X}^{x_{0} x_{T}}$ from $x_{0}$ to $x_{T}$ is given by

$$
\begin{aligned}
d \mathbf{X}^{x_{0} x_{T}}= & \left(-\nabla E\left(\mathbf{X}^{x_{0} x_{T}}\right)+\sigma \sigma^{T} \nabla \log p\left(\mathbf{x}_{T}, T \mid \mathbf{X}^{x_{0} x_{T}}, t\right)\right) d t \\
& +\sigma d \mathbf{W},
\end{aligned}
$$

where $p(\mathbf{y}, u \mid \mathbf{x}, t)$ denotes the transition density of $\mathbf{X}$, see [7]. In general, the transition density is unknown, which makes sampling from the bridge process challenging, in particular in high-dimensional spaces. There is a rather extensive literature on the sampling of diffusion bridges that work quite well in low dimensional spaces, including [8, 11, 10, 5, 3, 26].

The situation is greatly simplified if the distribution $p$ on $\mathbb{R}^{d}$ is of product form: $p(x)=\prod_{i=1}^{d} p_{i}\left(x_{i}\right) \propto \prod_{i=1}^{d} e^{E_{i}\left(x_{i}\right)}$. In that case each coordinate can be treated separately and $\mathbf{X}(t)=\left(X_{1}(t), \ldots, X_{d}(t)\right)$ with

$$
d X_{i}(t)=-\nabla E_{i}\left(X_{i}\right) d t+\sqrt{2} d W_{i}(t),
$$

has $p$ as its invariant distribution. The bridge process is simply obtained by considering the corresponding one-dimensional bridges.

\subsection{Standard normal diffusion bridge}

In the context of VAEs we consider stochastic processes in the latent space, denoted by $\mathbf{Z}=\{\mathbf{Z}(t)\}_{t \in[0, T]}$. If the prior $p(\mathbf{z})$ is an $n$-dimensional standard normal distribution,

$$
p(\mathbf{z})=(2 \pi)^{-n / 2} e^{-2^{-1} \mathbf{z z}^{T}}
$$

it follows that (2) reads

$$
d \mathbf{Z}=-\mathbf{Z} d t+\sqrt{2} d \mathbf{W}
$$


This process will henceforth be referred to as the standard normal diffusion process, which is a special case of an Ornstein-Uhlenbeck process. The corresponding bridge process will be referred to as the standard normal diffusion bridge process. Since $\mathbf{Z}$ has independent coordinates, the corresponding bridge process can be constructed from the coordinates individually, where each coordinate follows the one-dimensional process

$$
\begin{aligned}
d Z= & {\left[-Z+\frac{2 e^{-(T-t)}}{1-e^{-2(T-t)}}\left(z_{T}-Z e^{-(T-t)}\right)\right] d t } \\
& +\sqrt{2} d W
\end{aligned}
$$

with $Z(0)=z_{0}$. Implementing interpolation with (7) is as simple as replacing step (2) in Algorithm 1 with:

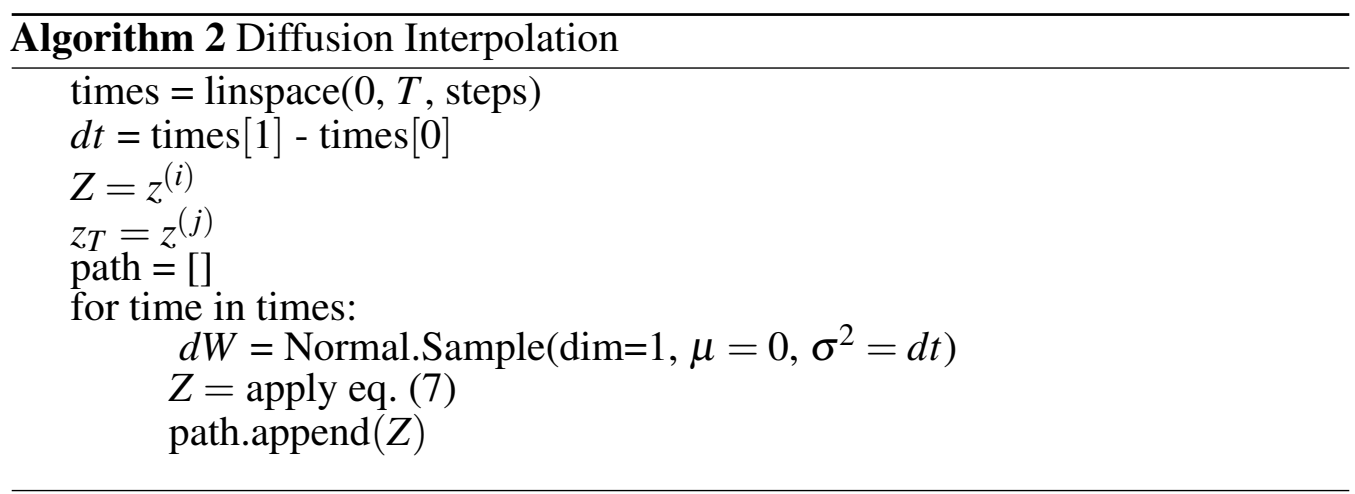

\subsection{Gaussian Processes}

Obviously, one is not restricted to use diffusion bridges to construct stochastic interpolation. In plain vanilla VAEs with normal priors it is also natural to consider bridges constructed from stationary Gaussian processes, with the prior as stationary distribution. Gaussian bridges are easy to construct and by adapting the covariance function makes it possible to control the properties, e.g. regularity and periodicity, of the interpolation paths. A stochastic process $\{Z(t)\}_{t \in \mathbb{R}}$ is a centered stationary Gaussian process if for any finite number of times $\left(t_{0}, \ldots, t_{m}\right)$, it holds that

$$
\left(Z\left(t_{0}\right), \ldots, Z\left(t_{m}\right)\right) \sim N(\mathbf{0}, \Sigma)
$$

$\Sigma$ is a positive semi-definite matrix, with $\Sigma_{i j}=k\left(t_{i}-t_{j}\right)$ for a symmetric kernel $k$. In this paper, the following kernels will be considered. For non-period sampling, the kernel 


$$
k(h)=\exp \left\{-\beta|h|^{\alpha}\right\}
$$

is used. With $\alpha=\beta=1$ it coincides with the covariance function of the standard normal diffusion. The parameter $\alpha$ controls the regularity of the paths of the Gaussian process, with higher $\alpha$ corresponding to smoother paths, whereas $\beta$ is a scale parameter. Indeed, since

$$
k(h)=1-\beta|h|^{\alpha}+o\left(|h|^{\alpha}\right),
$$

by the Kolmogorov-Chentsov conditions, the paths are almost surely Hölder continuous of all orders less than $\alpha / 2$. For periodic sampling, the kernel

$$
k(h)=\exp \left\{-\frac{2}{\ell^{2}} \sin ^{2}\left(\pi \frac{|h|^{2}}{p}\right)\right\}
$$

is considered. Note that $k(0)=1$ for any $t$, which implies that the stationary distribution has unit variance.

\subsection{Gaussian bridges}

The distributional structure of the Gaussian process allows for an easy construction of the Gaussian bridge using the conditional distribution of multivariate normal distributions.

Proposition 1. Let $\{Z(t)\}_{t \in \mathbb{R}}$ be a centered stationary Gaussian process with covariance kernel $k, E[Z(t) Z(s)]=k(t-s)$. Let $\left\{Z^{z_{0} z_{T}}(t)\right\}_{t \in[0, T]}$ be the Gaussian bridge obtained by conditioning $Z$ on $Z(0)=z_{0}$ and $Z(T)=z_{T}$. Then, $Z^{z_{0} z_{T}}$ is a Gaussian process with mean function $\hat{\mu}_{z_{0}, z_{T}}$ and covariance $\hat{k}$ given by

$$
\begin{aligned}
\hat{\mu}_{z_{0}, z_{T}}(t)= & \frac{z_{0}[k(t)-k(T-t) k(T)]+z_{T}[k(T-t)-k(t) k(T)]}{1-k(T)^{2}} \\
\hat{k}(t, s)= & k(t-s)+\frac{k(T)[k(T-s) k(t)+k(s) k(T-t)]}{1-k(T)^{2}} \\
& -\frac{k(T-s) k(T-t)+k(s) k(t)}{1-k(T)^{2}} .
\end{aligned}
$$

The proof follows by considering the joint Gaussian distribution of

$$
\left(Z(0), Z\left(t_{1}\right), \ldots, Z\left(t_{m}\right), Z(T)\right)
$$

conditioning on $(Z(0), Z(T))$, and using the properties of conditional Gaussian distributions. The proof follows by considering the joint Gaussian distribution of $\left(Z(0), Z\left(t_{1}\right), \ldots, Z\left(t_{m}\right), Z(T)\right)$, conditioning on $(Z(0), Z(T))$, and using the properties of conditional Gaussian distributions. 
Implementing interpolation with Gaussian Bridges amounts to replacing step (2) in Algorithm 1 with:

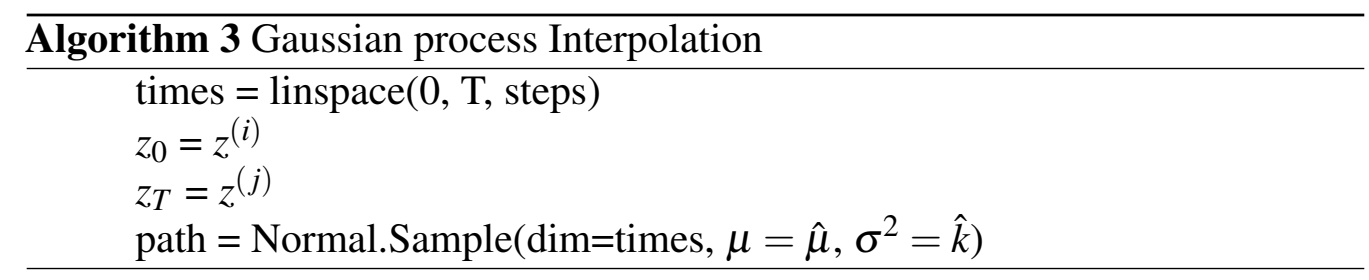

\section{Deviations from the typical set}

As discussed in the introduction, in the context of trained VAEs, encoded datapoints will typically reside in areas of high prior distribution mass, and the decoder will produce meaningful output only from these areas. Note that this does not necessarily imply that the likelihood of encoded individual data points $p\left(z^{i}\right)$ is high, which, in the case of standard normal priors would mean that encoded data points are close to the origin. In contrast, the asymptotic equipartition principle implies that, in high dimension $n$, the mass of a standard normal distribution is concentrated near the typical set:

$$
\begin{aligned}
S_{n \gamma} & =\left\{\left(z_{1}, \ldots, z_{n}\right) \in \mathbb{R}^{n}:\left|-n^{-1} \sum_{i=1}^{n} \log \phi\left(z_{i}\right)-H\right|<\gamma\right\} \\
& =\left\{\left(z_{1}, \ldots, z_{n}\right) \in \mathbb{R}^{n}:\left|n^{-1} \sum_{i=1}^{n} z_{i}^{2}-1\right|<2 \gamma\right\}, \quad \gamma>0,
\end{aligned}
$$

where $\phi$ denotes the standard normal density and $H$ its entropy. That is, the typical set is a thin sphere of radius $\sqrt{n}$. Below we provide theoretical support that, when $n$ is large, Gaussian bridges generate paths that reside in the vicinity of the sphere or radius $\sqrt{n}$. These results may be applied to select the length $T$ of the interpolation paths. To this end, first select the typical set $S_{n \gamma}$ by setting $\gamma>0$ so that $P\left(\left(Z_{1}, \ldots, Z_{n}\right) \in S_{n \gamma}\right)$ is high. For example, with $n=100$ a straightforward calculation yields that for $\gamma_{.95}=0.138$ the probability of the typical set is roughly 0.95 . Next we investigate to what extent the normalised squared radius of a Gaussian bridge

$$
R_{n}^{2}(t)=\frac{\sum_{i=0}^{n-1} Z_{i}^{z_{i} z_{i T}}(t)^{2}}{n}
$$

takes values in the typical set, i.e. $R_{n}^{2}(t) \in\left(1-2 \gamma_{.95}, 1+2 \gamma_{.95}\right)$. Here, $Z_{i}^{z_{i 0} z_{i T}}$ denotes the $i$ th coordinate process of a centered stationary Gaussian process with 
unit variance, conditioned on starting at $z_{i 0}$ and ending at $z_{i T}$. The following proposition provides a closed form approximation of (13) in high dimension.

Proposition 2. Let $\lambda_{n}^{0}=n^{-1} \sum_{i=1}^{n} \delta_{z_{i 0}}$ and $\lambda_{n}^{T}=n^{-1} \sum_{i=1}^{n} \delta_{z_{i T}}$ and suppose that $\lambda_{n}^{0} \rightarrow \lambda^{0}$ and $\lambda_{n}^{T} \rightarrow \lambda^{T}$ weakly, as $n \rightarrow \infty$, and

$$
\sup _{n} \int z^{2} \lambda_{n}^{0}(d z)<\infty, \quad \sup _{n} \int z^{2} \lambda_{n}^{T}(d z)<\infty .
$$

Let $Z^{\lambda_{0} \lambda_{T}}$ be a Gaussian bridge based on the kernel $k$, with $Z^{\lambda_{0} \lambda_{T}}(0) \sim \lambda^{0}$ and $Z^{\lambda_{0} \lambda_{T}}(T) \sim \lambda^{T}$. Then, for each $t \in[0, T]$,

$$
\begin{aligned}
& \lim _{n \rightarrow \infty} R_{n}^{2}(t)=E\left[Z^{\lambda_{0} \lambda_{T}}(t)^{2}\right] \\
& =\hat{k}(t, t)+\iint \hat{\mu}_{z_{0}, z_{T}}(t)^{2} \lambda^{0}\left(d z_{0}\right) \lambda^{T}\left(d z_{T}\right),
\end{aligned}
$$

with $\hat{\mu}$ and $\hat{k}$ as in Proposition 1.

Proof. Let $\{Z(t)\}_{t \in \mathbb{R}}$ be a centered stationary Gaussian process with kernel $k$. By the strong law of large numbers

$$
\frac{1}{n} \sum_{i=1}^{n}\left(Z_{i}^{z_{i 0} z_{i T}}(t)^{2}-E\left[Z_{i}^{z_{i 0} z_{i T}}(t)^{2}\right]\right) \rightarrow 0
$$

almost surely, where we have the representation

$$
\begin{aligned}
& \frac{1}{n} \sum_{i=1}^{n} E\left[Z_{i}^{z_{i 0} z_{i T}}(t)^{2}\right] \\
& =\frac{1}{n} \sum_{i=1}^{n} E\left[Z(t)^{2} \mid Z(0)=z_{i 0}, Z(T)=z_{i T}\right] \\
& =\iint E\left[Z(t)^{2} \mid Z(0)=z_{0}, Z(T)=z_{T}\right] \lambda_{n}^{0}\left(d z_{0}\right) \lambda_{n}^{T}\left(d z_{T}\right)
\end{aligned}
$$

The integrand is

$$
E\left[Z(t)^{2} \mid Z(0)=z_{0}, Z(T)=z_{T}\right]=\hat{k}(t, t)+\hat{\mu}_{z_{0}, z_{T}}(t)^{2} .
$$

Since it is continuous in $\left(z_{0}, z_{T}\right)$ it follows by weak convergence of $\lambda_{n}^{0} \otimes \lambda_{T}^{n} \rightarrow$ $\lambda^{0} \otimes \lambda^{T}$ and the integrability conditions, that the expression in the last display converges to

$$
\begin{aligned}
& \iint E\left[Z(t)^{2} \mid Z(0)=z_{0}, Z(T)=z_{T}\right] \lambda^{0}\left(d z_{0}\right) \lambda^{T}\left(d z_{T}\right) \\
& =\hat{k}(t, t)+\iint \hat{\mu}_{z_{0}, z_{T}}(t)^{2} \lambda^{0}\left(d z_{0}\right) \lambda^{T}\left(d z_{T}\right) .
\end{aligned}
$$


This completes the proof. In addition to the limiting behavior of the normalised radius, it is also straightforward to obtain point-wise prediction intervals for the normalised squared radius of the interpolation paths. Indeed, the mean and variance of $R_{n}^{2}(t)$ may be computed as

$$
E\left[R_{n}^{2}(t)\right]=\hat{k}(t, t)+\frac{1}{n} \sum_{i=1}^{n} \hat{\mu}_{z_{i 0}, z_{i T}}(t)
$$

and

$$
\begin{aligned}
\operatorname{Var}\left[R_{n}^{2}(t)\right] & =\frac{1}{n^{2}} \sum_{i=1}^{n} \operatorname{Var}\left[Z_{i}^{z_{i 0} z_{i T}}(t)^{2}\right] \\
& =\frac{1}{n^{2}} \sum_{i=1}^{n} \mathrm{E}\left[Z_{i}^{z_{i 0} z_{i T}}(t)^{4}\right]-\mathrm{E}\left[Z_{i}^{z_{i 0} z_{i T}}(t)^{2}\right]^{2} \\
& =3 \hat{k}(t, t)^{4}+\frac{6 \hat{k}(t, t)^{2}}{n^{2}} \sum_{i=1}^{n} \hat{\mu}_{z_{i 0}, z_{i T}}(t)^{2} .
\end{aligned}
$$

Figure 1 illustrates the mean $E\left[R_{n}^{2}(t)\right]$ and prediction interval $E\left[R_{n}^{2}(t)\right] \pm$ $\sqrt{\operatorname{Var}\left(\mathrm{R}_{\mathrm{n}}^{2}(\mathrm{t})\right)}$, which gives some insight into the typical whereabouts of the interpolation paths for different dimensions, $n$. In Figure 1 the starting- and end-point are perpendicular, the starting-point residing inside the sphere and the end-points residing outside of the sphere. The kernel (9) is used, with parameters $\alpha=2, \beta=2$. As can be observed, the interpolation paths respect the prior in that it resides near the typical set for all dimensions. For high dimensions, the interpolation path indeed stays close to the sphere for a considerable time before heading out to its end-point. In lower dimensions, although the expected trajectory is the same, the standard deviation interval suggests interpolation paths are frequently traversing the interior (and exterior) of the sphere.

\subsubsection{The choice of $T$}

Consider a Gaussian bridge process of high dimension $n$, constructed from a centered stationary Gaussian process with kernel $k$. Suppose, for simplicity that all coordinate processes start in 1 and end in -1 . Note that this process start and end on the opposite poles of the sphere of radius $\sqrt{n}$. Thus the expected normalised radius $E\left[R_{n}^{2}(t)\right]=\hat{k}(t, t)+\hat{\mu}_{1,-1}(t)=: R^{2}(t)$ does not depend on $n$ and starts and ends at 1 . Since all start-point coordinates and end-point coordinates are identical, the function $R(t)$ can be used for analysing the normalised radius of the bridge. In Figure $1, R(t)$ is plotted for different $T$, with $z_{0}=1, z_{T}=-1$ and $k$ given by (9) with $\alpha=\beta=1$.

In Figure 2, it is observed that longer times allow the bridge to stay closer to the sphere. For $T=3$, the path stays in the vicinity of the sphere for all times up 


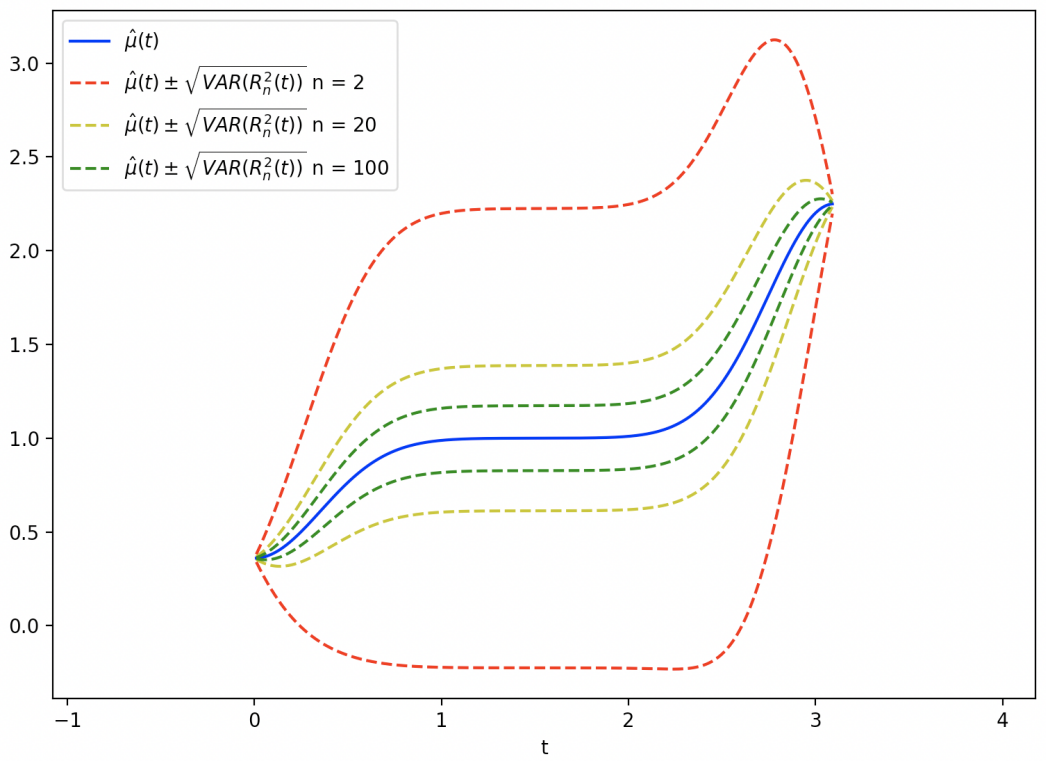

Fig. 1 Expected value of trajectory $\mathrm{E}\left[R_{n}(t)\right]$ (independent of $n$ ) in blue, together with prediction intervals (dependent of $n$ ), for different dimensions $n$. The starting points used are $[0.6 \sqrt{n}, 0,0, \ldots, 0]$ where the number of zeros are $n-1$. The end points used are $[0,1.5 \sqrt{n}, 0, \ldots, 0]$ where the number of zeros are $n-1$.
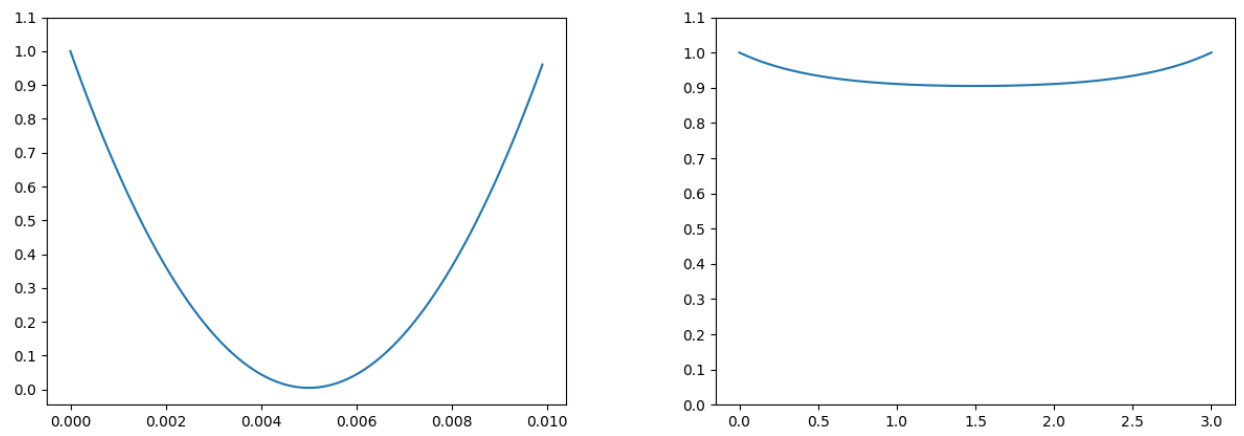

Fig. $2 R(t)$ plotted for times $T=0.01$ (left) and $T=3$ (right) with coordinates starting in 1 and ending in -1 . The longer time the closer the path stays to the sphere.

to $T$. On the other extreme, for $T=0.01$ the path crosses a vicinity of the origin, with small prior mass. This is quite intuitive. When $T$ is large, the process does not have to "hurry" by taking the shortest (linear) path through areas of low mass in order to reach the end-point within the specified time. When time-pressure is removed, the process adheres to its tendency to follow the stationary distribution, which has mass around the sphere. 
With these arguments in mind, one can claim that a suitable choice for $T$ is the smallest possible $T$ so that the expected normalized radius $R^{2}(t)$ of the interpolation path stays within the typical set.

\section{Experiments}

In this section, experiments are performed on two data sets. First, although we emphasize that our proposed interpolation method is inherently stochastic, we demonstrate empirically, as argued for theoretically above; that our method may be adapted to exhibit similar characteristics as linear interpolation and spherical interpolation [31] respectively, depending on the dimension and the choice of interpolation time $T$. The similarity is demonstrated visually through videos and pictures and quantitatively by measuring the mean distance to data in latent space over interpolation paths of 20 randomly selected start/end-points, as suggested by [4]. Last, but most importantly, we demonstrate the benefits of the random property of our interpolation method.

\subsubsection{MNIST, low dimensionality}

The MNIST dataset [21] consists of hand-written digits 0-9, white with a black background. We use a VAE on the training subset of MNIST, with architecture as follows. Encoder: two dense layers with relu non-linearity, first 200 units, second 200 units (mapping to latent space without non-linearity). Latent variables: mean and log-variance of Gaussian, no non-linearity, 2 units. Decoder: Two dense layers with relu non-linearity, first 200 units, second 200 units (mapping to output without non-linearity, Bernoulli). Batch size: 100. Learning rate: 0.001. Number of epochs: 600. Optimizer: AdamOptimizer. It is indicated in Section 4 that for a sufficiently small $T$, our method yields a path similar to the linear path. To see this in an empirical setting, we choose two 'extreme' points $\left(z^{\text {start }}, z^{\text {end }}\right)$ from the test data of digits "0" and "1", respectively, in the latent space. As shown in Figure 3, our method generates an interpolation path that is similar to the linear path. Both our method and linear interpolation result in an image sequence which is a visually appealing interpolation between "0" and "1", whereas spherical interpolation, which would be the preferable strategy in a state space with high dimension, gives rise to middle steps in the interpolation that are not similar to any handwritten

digit. This occurs as the mass for lower dimensional Gaussian is concentrated in the center rather than on the sphere. The mean distance score confirms the visual inspection:

That is, our method is similar to linear interpolation, which should be preferable over spherical interpolation in this case, as it stays closer to the latent representations of data. 

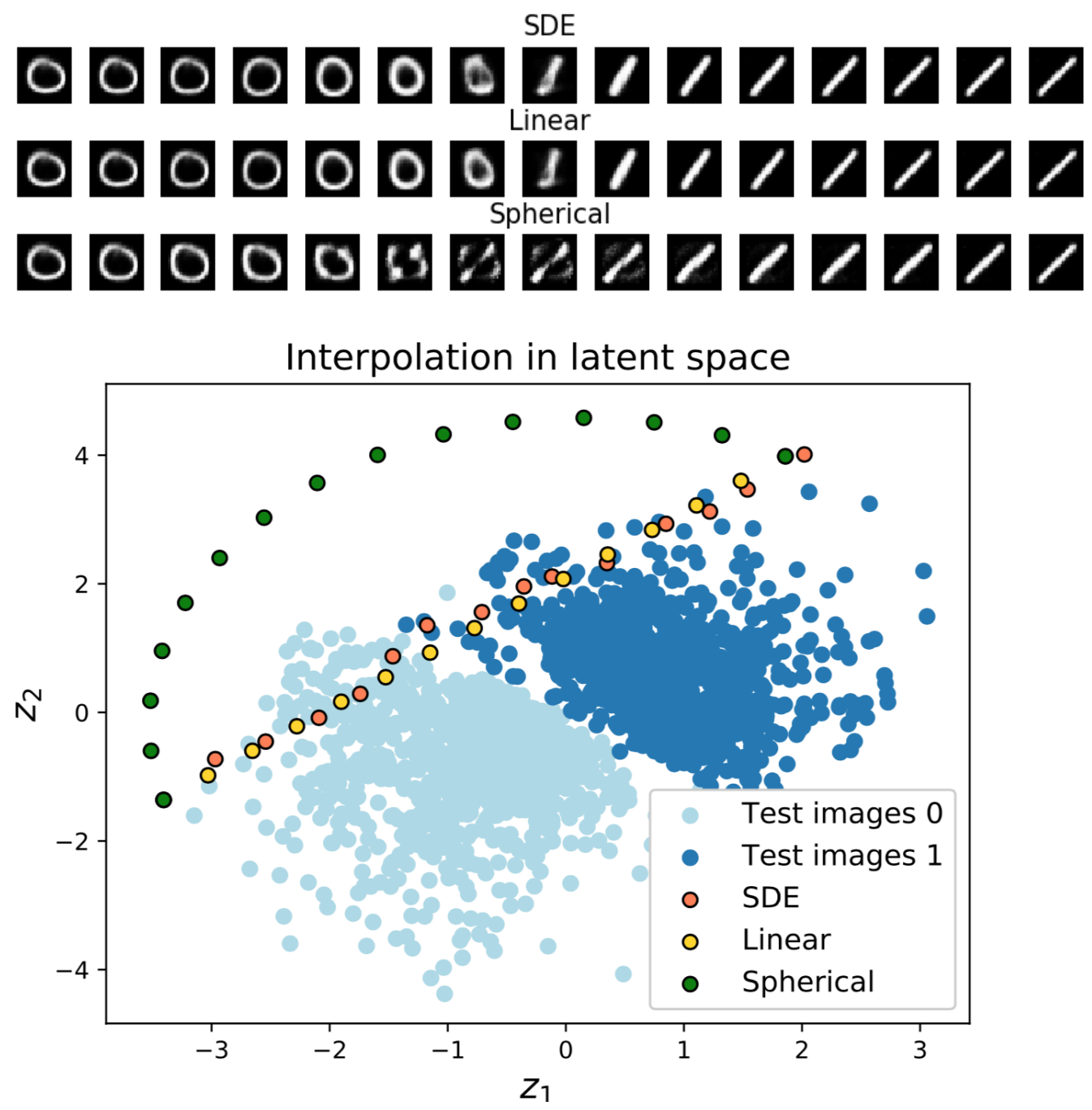

Fig. 3 Synthetic sequences using different interpolation methods, from a VAE trained on the MNIST dataset. A pair $\left(z^{\text {start }}, z^{\text {end }}\right)$ is chosen so as to demonstrate the disadvantages of spherical interpolation in low dimension. The top row and the red dots in the bottom plot show sequences generated through diffusion interpolation with $T=0.15$. The middle row and the yellow dots in the bottom plot show linear deterministic interpolation between the same points, and the bottom row and the green dots show deterministic spherical interpolation, again between the same points.

\subsubsection{Human3.6M, high dimensionality}

Human3.6M [17] consists of a large number of 3D motion capture sequences of human motion. Each time frame in the sequences consists of a list of $3 \mathrm{D}$ coordinates of body joint locations, which together describe the articulated pose of a human. We use a VAE implementation with architecture as follows. Encoder: Two dense layers with relu non-linearity, first 1000 units, second 500 units (mapping to latent 
Interpolation in Auto Encoders with Bridge Processes

\begin{tabular}{l|c} 
Method & Mean disctance score \\
\hline Linear & 0.284 \\
Spherical & 0.763 \\
Gaussian bridge & 0.280
\end{tabular}

Time

SDE
1)
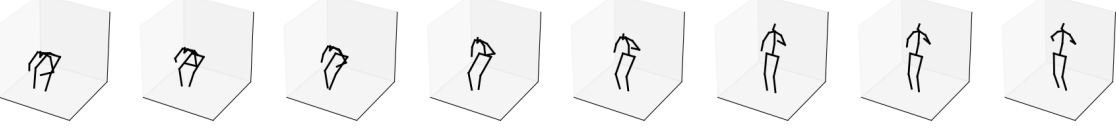
2) 专
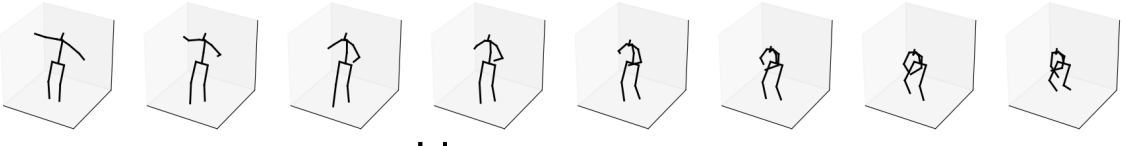
Linear
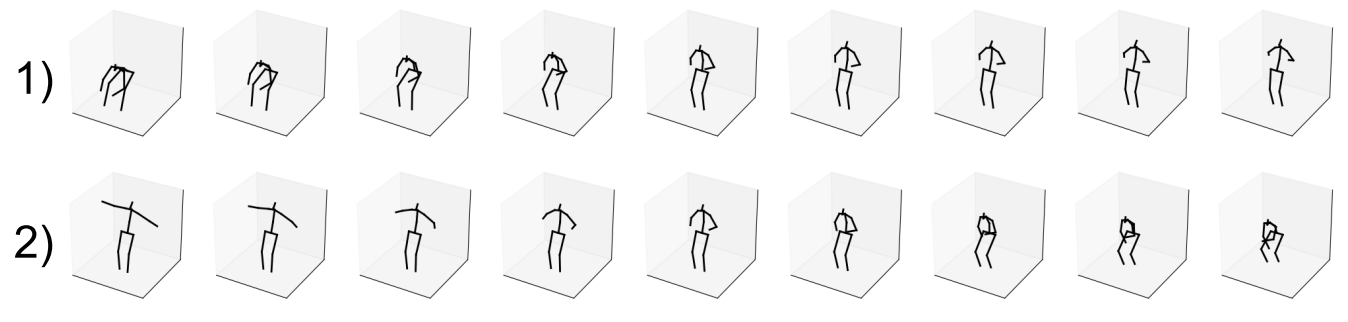

\section{Spherical}
1)
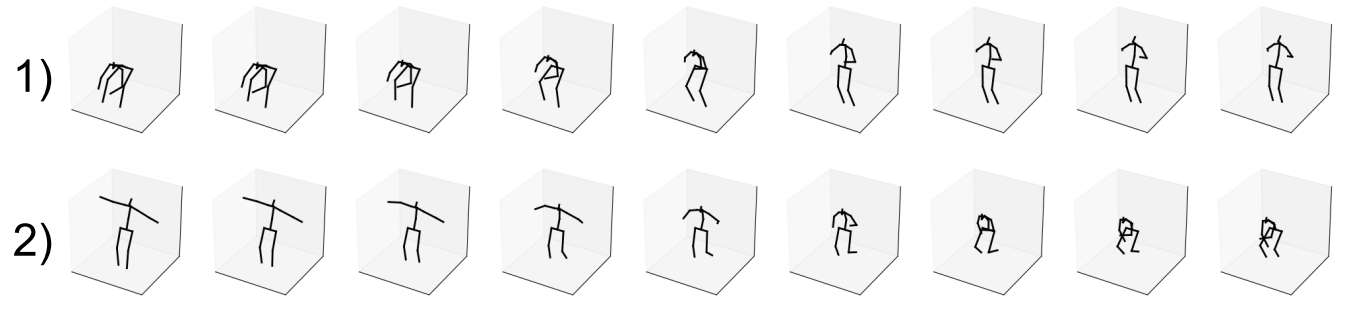

Fig. 4 Synthetic sequences using different interpolation methods, from the VAE trained on the Human3.6M dataset. Two pairs $\left(z_{1}^{\text {start }}, z_{1}^{\text {end }}\right)$ and $\left(z_{2}^{\text {start }}, z_{2}^{\text {end }}\right)$ were randomly sampled from the latent space. The top rows 1-2 show sequences generated through diffusion interpolation with $T=1$. The middle rows 1-2 show linear deterministic interpolation between the same points, and the bottom rows 1-2 show deterministic spherical interpolation, again between the same points.

space without non-linearity). Latent variables: mean and log-variance of Gaussian, no non-linearity, 30 units. Decoder: Two dense layers with relu non-linearity, first 500 units, second 1000 units (mapping to output without non-linearity). Batch size: 500. Learning rate: 0.001. Number of epochs: 10000 . Optimizer: Adagrad. Each pose is regarded as iid with respect to other poses in the sequence. Interpolation in the latent space will then correspond to generating a synthetic sequence from $z^{\text {start }}$ to $z^{\text {end }}$. 
Time

1)
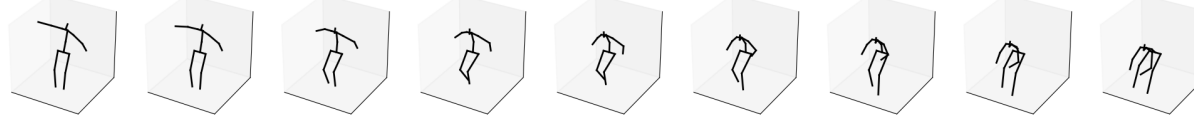

2)
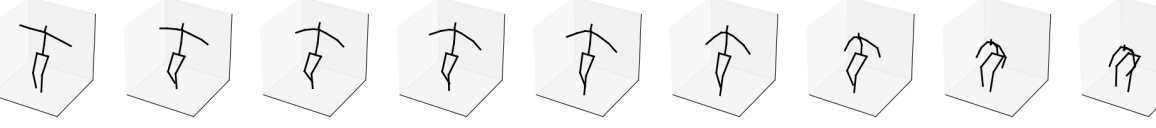

3)
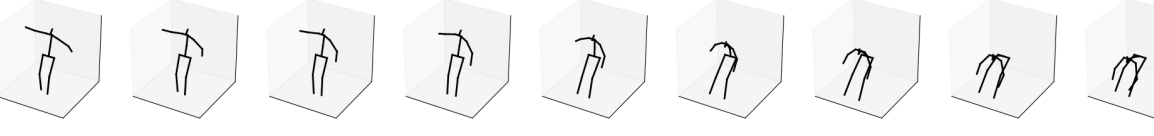

Fig. 5 Stochasticity in synthetic sequences using our approach, from the VAE trained on the Human3.6M dataset. A pair $\left(z^{\text {start }}, z^{\text {end }}\right)$ was randomly sampled from the latent space. Rows 1-3 depicts three different diffusion interpolations (stochastically varying) between these two points, with $T=1$.

As previously described, the preferable interpolation strategy for Gaussian priors of higher dimension $n$ is to move near the surface of a sphere around the origin with radius $\sqrt{n}$, as data is situated here with high probability for large enough dimension. It has been shown that our method stays close to the sphere of this radius given sufficient time $T$ and large enough dimension. Although linear interpolation in this specific high dimensional case is rather smooth and visually appealing, linear interpolation will pass near the origin in the vast majority of cases. This might not be desirable since nearly all interpolations thus will have similar midpoints.

Figure 4, middle rows, support this - linear interpolation between the two sampled pairs of start- and end-points both incorporate a part with the mean pose. This will of course be highly limiting if one would like to apply the method to synthetic motion generation.

On the other hand, a spherical interpolation (Figure 4, bottom rows) gives rise to natural-looking synthetic sequence without this constraint. We also see that our interpolation strategy (Figure 4, top rows) exhibit the same desirable feature.

The mean distance score,

\begin{tabular}{l|c} 
Method & Mean disctance score \\
\hline Linear & 5.10 \\
Spherical & 5.67 \\
Gaussian bridge & 5.28
\end{tabular}

shows that the three methods are very similar in performance in terms of vicinity to latent representations of data. Specifically, our method is similar to spherical interpolation, which should be preferable as it does not traverse the same midpoint for different start-end pairs to the same extent. 


\subsubsection{Random property demonstration, Human3.6M}

Here we illustrate the benefit of the stochastic property of our method (as opposed to deterministic interpolation). We emphasize that we regard this feature of our method as the main motivation for introducing it. Figure 5 shows three different interpolated sequences between the same start and end point. There are slight variations in the generated sequences, which is desirable if the method is used for motion synthesis or as a temporal prior for tracking. The effect becomes even clearer when observing videos. The following video links contain samples drawn from an interpolation with a periodic kernel (11), with $l^{2}=5, p=0.5, T=2.1$. Again, all interpolations starts and ends in the same latent points, are constructed with identical interpolation scheme; but each sample has clear distinguishing and natural features. We have given suitable names to the samples that characterises the walking style.

- Sample "struggling while walking":

https://youtu.be/BKErJVI2bSA=

- Sample "walking with confidence":

https://youtu.be/94-L5idkwJY=

- Sample "avoiding projectile while walking":

https://youtu.be/oPTB_GrJzPQ=.

\section{Conclusion}

A method is proposed for generating random interpolations in distributional latent space models, by considering bridge processes constructed from stationary stochastic processes whose stationary distribution is the prior distribution over the latent space. The setting is that of VAE models with normal priors, but the methodology is general and can be applied to any distributional latent space model. The proposed method enables generating different but realistic interpolations with each draw which is illustrated in the last videos, where realistic and clearly distinguishable videos of human walking are sampled through stochastic interpolation between two fixed points. The results can be applied to constructing priors for human tracking, or when sampling behaviour in a computer game, for example. The stochastic interpolation proposed here is not necessarily better than other deterministic interpolation methods in any particular metric. Rather, we argue, there are many possible interpolations that can conveniently be represented by a probability distribution over interpolation paths.

We demonstrate theoretically and empirically that the method has the desirable property of respecting the prior regardless of form and dimension. That is, the interpolation paths tend to lie in the typical set of the prior. In the case of VAE 
and normal priors, the proposed method is compared to two popular deterministic interpolation methods - linear interpolation and spherical interpolation. These methods are known to be good for low- and high-dimensional spaces, respectively. It is demonstrated that both of these methods can be viewed as deterministic special cases of our method, by varying the interpolation time.

The main limitation of the method is that it can be hard to construct bridges for general stochastic processes. There exist extensive literature on sampling diffusion bridges, but these are mainly concerned with low dimensional spaces. For high dimension and general prior it is not known to which extent the proposed method can be made practical.

\section{References}

1. E. Agustsson, A. Sage, R. Timofte, L.V Gool Optimal transport maps for distribution preserving operations on latent spaces of generative models. International Conference on Learning Representations, 2019.

2. M. Alamgir and U. von Luxburg. Shortest path distance in random k-nearest neighbor graphs. in Proc. 29th Int. Conf. on Machine Learning, 2012.

3. C. Bayer and J. Schoenmakers, J. Simulation of forward-reverse stochastic representations for conditional diffusions. Ann. Appl. Probab. 24(5), 19942032, 2014.

4. D. Berthelot, C. Raffel, A. Roy, and I. Goodfellow. Understanding and improving interpolation in autoencoders via adversarial regularizer. Preprint, arXiv:1807.07543, 2018.

5. A. Beskos, G. Roberts, A. Stuart and J. Voss, J. MCMC methods for diffusion bridges. Stoch. Dyn. 8(3), 319-350, 2008.

6. F.P. Casale, A.V. Dalca, L. Saglietti, J. Listgarten, N. Fusi Gaussian Process Variational Autoencoders Conference and Workshop on Neural Information Processing Systems, 2018.

7. U. Cetin and A. Danilova. Markov Bridges: SDE Representation Stochastic Processes and their Applications, 126(3):651-679, 2016.

8. J. Clark, J. The simulation of pinned diffusions. In Decision and Control, 1990., Proceedings of the 29th IEEE Conference on, 1418-1420. IEEE, 1990.

9. E. Davis and S. Sethuraman. Approximating geodesics via random points. Ann. Appl. Probab., 29(3), 1446-1486, 2019.

10. B. Delyon and Y. Hu. Simulation of conditioned diffusion and application to parameter estimation. Stochast. Process. Appl. 116(11), 1660-1675, 2006.

11. G.B. Durham and A.R. Gallant. Numerical techniques for maximum likelihood estimation of continuous-time diffusion processes. J. Bus. Econom. Statist. 20(3), 297-338, 2002.

12. D. Gasbarra, T. Sottinen and E. Valkeila. Gaussian bridges. In Stochastic analysis and applications, volume 2 of Abel Symp., 361-382, Springer, Berlin, 2007.

13. V. Fortuin, D. Baranchuck, G. Rätsch, and S. Mandt. Deep amortized variational inference for multivariate time series imputation with latent Gaussian process models. 2nd Symposium on Advances in Approximate Bayesian Inference, 1-15, 2019. 
14. P. Hall, J.S. Marron, and A. Neeman. Geometric representation of high dimension, low sample size data. Journal of the Royal Statistical Society: Series B (Stastistical Methodology), 67(3):427-444, 2005.

15. T.B. Hashimoto, Y. Sun, and T.S. Jaakkola. Metric recovery from directed un-weighted graphs. in Proc. 18th Int. Conference on AI and Stat (AISTATS), San Diego, CA., 2015

16. W.-N. Hsu, Y. Zhang, and J. Glass. Unsupervised learning of disentangled and interpretable representations from sequential data. In Advances in neural information processing systems, 1878-1889, 2017.

17. C. Ionescu, D. Papava, V. Olaru, and C. Sminchisescu. Human3.6M: Large scale datasets and predictive methods for 3D human sensing in natural environments. IEEE Transactions on Pattern Analysis and Machine Intelligence, 36(7):1312-1324, 2014.

18. I. Karatzas and S.E. Shreve. Brownian motion and stochastic calculus. 2nd Ed., Springer, 1991.

19. Y. Kilcher, A. Lucchi, and T. Hofmann. Semantic interpolation in implicit models. International Conference on Learning Representations, 2018.

20. D. Kingma and M. Welling. Auto-encoding variational Bayes. Preprint, arXiv:1312.6114, 2013.

21. Y. LeCun, L. Bottou, Y. Bengio, and P. Haffner. Gradient-based learning applied to document recognition. Proceedings of the IEEE, 86(11):2278-2324, 1998.

22. D. Lesniak, I. Sieradzki, and I. Podolak. Distribution-interpolation trade off in generative models. International Conference on Learning Representations, 2019.

23. Y. Li and S. Mandt. Disentangled sequential autoencoder. ICML, 2018.

24. D. Perrault-Joncas and M. Meilă. Directed Graph Embedding: an Algorithm based on Continuous Limits of Laplacian-type Operators NIPS, 2011.

25. D. Perrault-Joncas and M. Meilă. Estimating vector fields on manifolds and the embedding of directed graphs. arXiv:1406.0013v1, 2014.

26. M. Schauer, F. van der Meulen and H. van Zanten. Guided proposals for simulating multi-dimensional diffusion bridges. Bernoulli, 23(4A), 2917$2950,2015$.

27. R. Singh, P. Turaga, S. Jayasuriya, R. Garg, and M.W. Braun. Non-parametric priors for generative adversarial networks. International Conference on Machine Learning, 2019.

28. N. Srivastava, E. Mansimov, and R. Salakhutdinov. Unsupervised learning of video representations using lstms. Proceedings of the 32nd International Conference on International Conference on Machine Learning, 37, 843-852, 2015.

29. A.S. Sznitman. Topics in propagation of chaos. Ecole d'Eté de Probabilités de Saint-Flour XIX. Lecture Notes in Mathematics, vol 1464. Springer, Berlin, Heidelberg 1989.

30. M. Tschannen, O. Bachem, and M. Lucic. Recent advances in autoencoderbased representation learning. arXiv preprint arXiv:1812.05069, 2018.

31. T. White. Sampling generative network. Preprint, arXiv:1609.04468, 2016.

32. C. Ylidiz, M. Heinonen, and H. Lähdesmäki. ODE ${ }^{2}$ VAE: Deep generative second order ODEs with Bayesian neural networks. 33rd Conference on Neural Information Processing Systems (NeurIPS), 2019. 